\title{
SOME IMBEDDING THEOREMS AND CHARACTERIZATION PROBLEMS OF DISTANCE GEOMETRY
}

\author{
L. M. BLUMENTHAL
}

Introduction. The systematic development of abstract distance geometry was initiated by Menger's Untersuchungen [6] ${ }^{1}$ of 1928. The field opened up by these pioneering papers has been extensively cultivated during the past fourteen years with the result that not only have the boundaries of the subject been extended far beyond what was envisaged a decade and a half ago, but the territory gained has been regained and consolidated by new methods that attain their objectives more easily. It is the purpose of this paper to present some of these consolidations (Part I) and extensions (Part II) - at hand or in progress - and to point out a few promising regions for future exploration.

\section{PART I}

1. Preliminary definitions. A distance space, in its most general aspects, arises upon associating with each ordered pair $p, q$ of elements of a "point" set an element $p q$ of a "distance" set, the association being conditioned only by certain very simple rules. If, in particular, the distance set is the set of non-negative real numbers, $p q=q p$, while $p q=0$ if and only if $p=q$, one obtains the class $\{\Sigma\}$ of semimetric spaces. A semimetric space is metric provided that for each three of its points $p, q, r$ the triangle inequality $p q+q r \geqq p r$ is satisfied.

We shall have frequent occasion to consider the determinant

$$
D\left(p_{1}, p_{2}, \cdots, p_{k}\right)=\left|\begin{array}{ccccc}
0 & 1 & 1 & \cdots & 1 \\
1 & 0 & p_{1} p_{2}^{2} & \cdots & p_{1} p_{k}^{2} \\
1 & p_{2} p_{1}^{2} & 0 & \cdots & p_{2} p_{k}^{2} \\
\cdot & \cdot & \cdot & \cdots & \cdot \\
1 & p_{k} p_{1}^{2} & p_{k} p_{2}^{2} & \cdots & 0
\end{array}\right|
$$

formed for $k$ points $p_{1}, p_{2}, \cdots, p_{k}$ of a semimetric space. With its use the triangle inequality takes the symmetric form $D(p, q, r) \leqq 0$.

Two distance spaces (or subsets of the same distance space) are

An address delivered before the Chicago meeting of the Society on April 17, 1942, by invitation of the Program Committee; received by the editors May 25, 1942.

${ }^{1}$ See references at end of paper. 
called congruent or isometric provided there exists a one-to-one, distance-preserving correspondence between their points, and a distance geometry studies those properties of a distance space which are invariant under the group of congruences.

2. Problems for euclidean subsets and spaces. It is observed that a semimetric space of $m$ elements $p_{1}, p_{2}, \cdots, p_{m}$ is given by a matrix $\left(p_{i} p_{j}\right)$ of non-negative real numbers - the mutual distances of the $m$ points - which, in general, is subject only to the requirements of being symmetric and having zeros along the principal diagonal. It is clear that if such a distance matrix be formed for $m$ points of a particular space (for example, euclidean) there is a marked decrease in the freedom with which the elements of the matrix may be selected. The distinctive character of the space is reflected in the structure (the inner relations) of this matrix, and it is natural to seek to classify and study distance spaces in terms of this structure. ${ }^{2}$ The first problems to which these considerations gave rise were the following ones.

THE EUCLIDEAN SUBSET PROBLEM. What is the structure of the distance matrix of a finite semimetric space which is necessary and sufficient to insure the euclidean character of the metric? On what finite subsets (if any) of an arbitrary semimetric $\Sigma$ is it necessary and sufficient to impose this matrix structure in order that $\Sigma$ may be congruently contained in a euclidean space of a given dimension? ${ }^{3}$

ThE EUCLIDEAN SPACE PROBLEM. Let $\Sigma$ be any semimetric space. What are necessary and sufficient (metric) conditions for congruence of $\Sigma$ with a euclidean space of given dimension?

The first solutions of both of these problems were given by Menger [6].

It is important to observe that the subset problem is the more general one. Of the many solutions that have been given for the space problem few advance the solution of the problem of congruent imbedding. This seems due to the fact that in characterizing a space one

2 There are many ways in which this structure may be described. As seen later, one may base the description upon (1) the signs of principal minors of $D\left(p_{1}, \cdots, p_{m}\right)$ (Menger), (2) the character of the quadratic form with determinant $D$ (Morse), (3) the character of the quadratic form $(1 / 2) \sum_{2}^{m}\left(p_{1} p_{i}^{2}+p_{1} p_{j}^{2}-p_{i} p_{j}^{2}\right) x_{i} x_{j}$ (Schoenberg), (4) the behavior of the family of functions $\exp \left(-\lambda t^{2}\right), \lambda$ positive, defined over the space of $m$ elements (E. H. Moore, Schoenberg), and so on. It would be useful to add to these means of interpreting the structure.

${ }^{3}$ It should be mentioned that this formulation of the euclidean subset problem is quite different from the original one. 
imposes from the outset certain obvious necessary conditions that profoundly affect the type of theorem obtained. So far as imbeddability is concerned, such theorems give only stringent sufficient conditions.

Thus Busemann has shown that a convex, externally convex, finitely compact metric space is congruent to a euclidean or hyperbolic space provided the locus of points with equal distances from two distinct points contains with each distinct pair of its points each straight line through them. ${ }^{4}$ This interesting result is, however, of little use in determining if an arbitrary semimetric space is congruently contained in a euclidean or hyperbolic space.

On the other hand, a solution of the euclidean subset problem does advance the solution of the space problem, for if subsets of $E_{n}$ are characterized metrically a characterization of $E_{n}$ itself is obtained by adjoining properties that distinguish $E_{n}$ among its subsets. This is a euclidean rather than an abstract space problem. It should be mentioned that a characterization of the $E_{n}$ by way of the subset problem is quite likely to contain redundancies in the hypotheses, for it may happen that some of the requirements which are essential for the imbedding of arbitrary semimetric spaces in $E_{n}$ might be dispensed with in the light of those adjoined conditions individualizing the $E_{n}$ among its subsets. We shall see an illustration of this later.

Before turning to the solutions of the two problems posed above, we state here the general characterization problem.

GeNERAL CHARACTERIZATION PROBLEM. Let $\{\Delta\}$ be a given class of distance spaces and $\left\{\Delta^{*}\right\}$ a given subclass of $\{\Delta\}$. The subclass $\left\{\Delta^{*}\right\}$ is characterized metrically with respect to the class $\{\Delta\}$ when metric conditions are obtained which are necessary and sufficient to insure that any space of $\{\Delta\}$ satisfying them be congruent with a member of $\left\{\Delta^{*}\right\}$.

The problem of isometric or congruent imbedding consists in determining whether a given space $\Delta$ be congruently contained in a given space $\Delta^{*}$ (that is, congruent with a subset of $\Delta^{*}$ ). The congruence of the spaces $L^{(2)}$ and $l^{(2)}(1907)$, the isometric imbedding in Urysohn's space $U$ (or the space $C$ of continuous functions on $(0,1)$ ) of each separable metric space are examples.

3. Solution of euclidean subset and space problems. The problem of congruent imbedding of $\Sigma$ in $E_{n}$ is reduced to a "finite" one by

\footnotetext{
${ }^{4}$ For definitions of convexity, external convexity, and so on, see the writer's $D i s$ tance geometries, University of Missouri Studies, vol. 13 (1938), which presents a survey of the development of abstract metrics.
} 
showing that any semimetric space is congruently contained in $E_{n}$ whenever each $n+3$ of its points are. This is described by saying that the $E_{n}$ has congruence order $n+3$ with respect to the class of semimetric spaces. In the following we give the imbedding theorems with weaker hypotheses than those originally imposed. ${ }^{5}$

The congruence order $n+3$ property of the $E_{n}$ is a consequence of the following easily proved theorem:

THEOREM 3.1. A semimetric $\Sigma$ is congruently contained in $E_{n}$ if and only if there exists an integer $r, r \leqq n$, such that (1) $\Sigma$ contains $r+1$ points congruently imbeddable in $E_{r}$, not in $E_{r-1}$, and $(2)$ each $(r+3)$ tuple of $\Sigma$ containing this $(r+1)$-tuple is imbeddable in $E_{r}$. Then $\Sigma$ is congruently contained in $E_{r}$, not in $E_{r-1}$.

This theorem differs principally from Menger's congruence order theorem by not assuming that all $(r+3)$-tuples are imbeddable in $E_{r}$, but only those that contain a selected set of $r+1$ points. It thus permits the presence of free $(r+3)$-tuples; that is, sets of $r+3$ points which are not assumed imbeddable in $E_{r}$. We shall meet with other instances of this kind of freedom later. Further, the proof of the property as formulated above dispenses with a separation into cases and an induction which feature Menger's proof.

The euclidean subset problem is then solved when conditions for (1) the imbedding of $r+1$ points in $E_{r}$, not in $E_{r-1}$, and (2) the imbedding in $E_{r}$ of $(r+3)$-tuples containing such a set of $r+1$ points are obtained. Concerning (1) we have this statement:

Theorem 3.2. A semimetric $(r+1)$-tuple $p_{1}, p_{2}, \cdots, p_{r+1}$ is imbeddable in $E_{r}$, not in $E_{r-1}$, if and only if

$$
\operatorname{sgn} D\left(p_{1}, p_{2}, \cdots, p_{k+1}\right)=(-1)^{k+1}, \quad k=1,2, \cdots, r .
$$

This theorem was originally proved by Menger under the heavier assumptions that for each integer $k(2 \leqq k \leqq r+1)$ and for each set of $k$ of the $r+1$ points, sgn $D\left(p_{i_{1}}, p_{i_{2}}, \cdots, p_{i_{k}}\right)=(-1)^{k}$. Menger later observed that as a consequence of a remark of M. Morse it suffices to require merely the non-vanishing of the determinants of every $k$ tuple $(2 \leqq k \leqq r+1)$ of the points and $\operatorname{sgn} D\left(p_{1}, p_{2}, \cdots, p_{k+1}\right)$ $=(-1)^{k+1}(k=1,2, \cdots, r)[5]$. It turns out, however, that even the non-vanishing of these determinants need not be assumed. ${ }^{6}$

${ }^{5}$ In a recent seminar course, the writer has completely revised Menger's treatment of the euclidean problems and obtained simpler proofs and stronger theorems. This applies particularly to the difficult matter of quasi-congruence order.

${ }^{6}$ This is implied also by Schoenberg's quadratic form criterion, but we shall use these results to obtain Schoenberg's theorem. 
Corollary. If $p_{1}, p_{2}, \cdots, p_{r}$ are congruently contained in $E_{r-1}$, not in $E_{r-2}$, then the semimetric $(r+1)$-tuple $p_{1}, p_{2}, \cdots, p_{r}, p_{r+1}$ is imbeddable in $E_{r}$, not in $E_{r-1}$, if and only if $\operatorname{sgn} D\left(p_{1}, p_{2}, \cdots, p_{r}, p_{r+1}\right)$ $=(-1)^{r+1}$.

It is of interest that the inequality demanded of the determinant $D$ of the $r+1$ points is strong enough to force the selection of the $r$ distances of $p_{r+1}$ from the remaining $r$ points so that the $r$-tuples $p_{1}, \cdots, p_{i-1}, p_{i+1}, \cdots, p_{r}, p_{r+1}(i=1,2, \cdots, r)$ are euclidean. Turning to the imbedding of $r+3$ points, we have this theorem.

Theorem 3.3. If $p_{1}, \cdots, p_{r+1}$ are imbeddable in $E_{r}$, not in $E_{r-1}$, then the semimetric $(r+3)$-tuple $p_{1}, \cdots, p_{r+1}, p_{r+2}, p_{r+3}$ is imbeddable in $E_{r}$ if and only if

$$
\begin{aligned}
D\left(p_{1}, \cdots, p_{r+1}, p_{r+2}\right) & =D\left(p_{1}, \cdots, p_{r+1}, p_{r+3}\right) \\
& =D\left(p_{1}, \cdots, p_{r+1}, p_{r+2}, p_{r+3}\right)=0 .
\end{aligned}
$$

The proof of this theorem follows familiar lines when it is established that $p_{1}, p_{2}, \cdots, p_{r+1}, p_{r+2}$ and $p_{1}, p_{2}, \cdots, p_{r+1}, p_{r+3}$ are imbeddable in $E_{r}$, not in $E_{r-1}$. This is done in a lemma, and the euclidean subset problem is completely solved. ${ }^{7}$

By despoiling $D\left(p_{0}, p_{1}, \cdots, p_{k}\right)$ of its bordering, we have

$$
D\left(p_{0}, p_{1}, \cdots, p_{k}\right)=(-1)^{k+1} \cdot 2^{k}\left|p_{i j}\right|, \quad i, j=1,2, \cdots, k,
$$

where $p_{i j}=(1 / 2)\left(p_{0} p_{i}^{2}+p_{0} p_{j}^{2}-p_{i} p_{j}^{2}\right)$. It follows at once from the preceding theorems that a semimetric $(n+1)$-tuple $p_{0}, p_{1}, \cdots, p_{n}$ is imbeddable in $E_{n}$ if and only if there exists an integer $r, r \leqq n$, such that for $r+1$ of the $n+1$ points, say $p_{0}, p_{1}, \cdots, p_{r},\left|p_{i j}\right|>0$ $(i, j=1,2, \cdots, k ; k \leqq r)$ while in case $r<n$, all $(r+1)$ st and $(r+2) \mathrm{d}$ order determinants $\left|p_{i j}\right|$ containing $\left|p_{i j}\right|(i, j=1,2, \cdots, r)$ vanish. These are precisely the conditions that the quadratic form $\sum_{i, j=1}^{n} p_{i j} x_{i} x_{j}$ be positive definite of rank $r$, and we have the theorem of Schoenberg [10]. It is easy to see that this condition is equivalent to the form $\sum_{i, j=0}^{n}\left(p_{i} p_{j}\right)^{2} x_{i} x_{j}$ being negatively definite of rank $r$ on the hyperplane $x_{0}+x_{1}+\cdots+x_{n}=0$, and this form of the condition has proved to be very useful.

Turning again to the congruence order $n+3$ property of the $E_{n}$, it

${ }^{7}$ We see that if $p_{1}, \cdots, p_{r}$ are imbeddable in $E_{r-1}$, not in $E_{r-2}$, then the semimetric $(r+1)$-tuple $p_{1}, \cdots, p_{r}, p_{r+1}$ is imbeddable in $E_{r}$ if and only if the sign of their determinant $D$ is not $(-1)^{r}$. An example shows this is no longer valid if the restriction that the $r$ points be not imbeddable in $E_{r-2}$ is dropped; for if $p q=q r=p s=1, p r=2$, $q s=3, r s=(19)^{1 / 2}$, the triple $p, q, r$ is imbeddable in $E_{2}$ and $D(p, q, r, s)=0$, but the four points are not congruently contained in a euclidean space. 
is natural to inquire into the conditions that permit a reduction in the number $n+3$. That this number is in general the smallest integer $k$ for which the imbedding of each $k$-tuple of a semimetric space implies the imbedding of the whole space in $E_{n}$ follows, as Menger has shown, from the existence for each integer $n$ of semimetric spaces of $n+3$ points which are not imbeddable in $E_{n}$ though each $(n+2)$-tuple is.

The construction of these so-called pseudo- $E_{n}(n+3)$-tuples is readily described, but only in the case of pseudo-linear quadruples $(n=1)$ have distance relations characterizing such sets been obtained. These quadruples have the form $p q=r s=a>0, q r=p s=b>0$, $p r=q s=a+b$, and $D(p, q, r, s)=-32$ (product of the six distances). It would be of interest to find the value of $D$ for a pseudo- $E_{n}(n+3)$ tuple. So far it has been shown only that its sign is $(-1)^{n}$.

It follows from the preceding remark that pseudo- $E_{n}$ sets of $n+3$ points are not imbeddable in any euclidean (or Hilbert) space. Pseudo-linear quadruples are clearly imbeddable in a (convex) circle, but pseudo-planar quintuples are not imbeddable, so far as is known, in any singularity-free surface. ${ }^{8}$ Recently spaces were constructed by Pepper which contain congruently all pseudo- $E_{n}(n+3)$-tuples [7]. They are obtained by appropriately metrizing the union of two $n$ spaces "joined" along a simplex. For $n=2$ a simpler kind of space suffices. This is obtained by metrizing convexly the set consisting of the union of three half-planes with a common axis.

What of pseudo- $E_{n}$ sets of more than $n+3$ points? The most complicated part of the Zweite Untersuchung is devoted to showing that no such sets exist; that is, if a semimetric space has more than $n+3$ points it is imbeddable in $E_{n}$ whenever each $n+2$ of its points has this property. This is described by saying that the $E_{n}$ has quasi-congruence order $n+2$ with respect to the class of semimetric spaces. We shall return to this matter later.

Having solved the euclidean subset problem, the characterization of the whole $E_{n}$ is obtained by adjoining properties distinguishing it from its subsets. This yielded the following theorem.

Theorem 3.4. A semimetric space $\Sigma$ is congruent to $E_{n}$ if and only if $\Sigma$ is complete, convex, externally convex, has each $n+2$ of its points imbeddable in $E_{n}$ and at least one $(n+1)$-tuple not imbeddable in $E_{n-1}$.

With this the principal objectives of the Zweite Untersuchung are attained. The reader is referred to Distance geometries for a discussion

\footnotetext{
${ }^{8}$ The convex circle is the circle (circumference) with shorter arc metric.
} 
of the results of $\mathrm{W}$. A. Wilson who, attacking the space problem directly, obtained a characterization theorem in terms of the imbedding of quadruples instead of $(n+2)$-tuples, as well as for the work of Aronszajn bearing upon the space problem. ${ }^{9}$ By weakening Wilson's four-point property to require merely that each quadruple containing a linear triple be imbeddable (weak four-point property) the writer allowed the existence of free quadruples. Very recently B. E. Gillam characterized the $E_{3}$ by showing that a complete, convex, externally convex metric space with the weak four-point property, which contains at least one quadruple with non-vanishing determinant $D$, while $D$ vanishes for each five points of the space, is logically equivalent to $E_{3}$ [4]. This was accomplished by proving all of Hilbert's postulates for $E_{3}$ on the basis of the above assumptions, with lines, planes, betweenness and congruence appropriately defined.

\section{PART II}

Foreword. As indicated in the introduction, this part of the paper is primarily concerned with extensions rather than consolidations. I propose to take up some recently established imbedding and characterization theorems, along with pertinent notions and concepts, and to raise certain questions suggested by them. No attempt is made to catalogue all of the new results; on the contrary, I limit myself to the discussion of the very few with which I have been connected.

1. Concerning quasi-congruence order and free $m$-tuples. We have seen that one of the important metric properties of the $E_{n}$ is its possession of quasi-congruence order $n+2$ with respect to semimetric spaces; that is, any semimetric space of more than $n+3$ distinct points is congruently contained in the $E_{n}$ whenever each $n+2$ of its points are. Several kinds of inquiries are suggested by this interesting concept.

(I). It has been observed in Part I that the requirement featuring the notion of congruence order $n+3$ (namely, that each $(n+3)$-tuple be imbeddable in $E_{n}$ ) has been weakened by allowing the presence of free $(n+3)$-tuples (that is, $(n+3)$-tuples which are not assumed congruently imbeddable in $E_{n}$ ) and the question arises whether a similar kind of weakening might be allowable here. Is the imbedding still valid if a certain number of the $(n+2)$-tuples are free? If so, how many of the $(n+2)$-tuples might be taken as free?

${ }^{9}$ It should be noted, however, that Wilson's work is not entirely independent of the subset problem for it utilizes Menger's theorem on the imbedding of $n+1$ points in $E_{n}$. 
In answering these questions it is first of all clear that it does not suffice to require merely that each $(n+2)$-tuple containing a selected set of $n+1$ independent points be imbeddable in $E_{n}$-a type of weakening immediately suggested by what proved to be effective in the case of congruence order. If $n=1$, for example, this demands only that every triple containing a given pair of distinct points be linear (that is, imbeddable in $E_{1}$ ). That the linearity of a space does not follow from this demand is seen by considering the convex circle, which is not imbeddable in the $E_{1}$ though each of its triples containing a given pair of diametral points is linear. There is, however, an analogue of the previous type of weakening. It is given in the following theorem.

THEOREM 1.1. Let $\Sigma$ be a semimetric space of at least $n+4$ distinct points. $\Sigma$ is congruently contained in the $E_{n}$, not the $E_{n-1}$, if and only if (a) $\Sigma$ contains a set $S$ of $n+2$ distinct points of which at least one $(n+1)$ tuple is not imbeddable in $E_{n-1}$, and (b) each $n+2$ points of $\Sigma$ with at least $n$ points in common with $S$ is imbeddable in $E_{n}$.

Proof. Let $p_{1}, p_{2}, \cdots, p_{n+1}, p_{n+2}$ denote the points of $S$, with the first $n+1$ not imbeddable in $E_{n-1}$. Using (b) it follows that these $n+1$ points are imbeddable in $E_{n}$. If, now, $p_{1}, p_{2}, \cdots, p_{n+1}, r, s$ is any $(n+3)$-tuple of distinct points containing the independent $n+1$ points, consider the $n+4$ points formed by annexing the point $p_{n+2}$. (The case where either $r$ or $s$ is $p_{n+2}$ causes no difficulty.) By (b) each $n+2$ of these $n+4$ points is congruently contained in $E_{n}$, and hence, by the quasi-congruence order $n+2$ property of $E_{n}$, the $n+4$ points are imbeddable in $E_{n}$. Thus each $(n+3)$-tuple containing the independent $(n+1)$-tuple $p_{1}, p_{2}, \cdots, p_{n+1}$ is imbeddable in $E_{n}$ and it follows that the whole space is congruently contained in $E_{n}$.

An example shows that this theorem is no longer valid if the assumption that at least one $(n+1)$-tuple of $S$ be not imbeddable in $E_{n-1}$ is not made, suppressing at the same time, of course, the requirement that $\Sigma$ be not imbeddable in $E_{n-1}$. Let $p_{1}, p_{2}, p_{3}$, form a plane equilateral triple and label the in-center $p_{4}$ and $p_{5}$. The semimetric space $\Sigma$ whose points are $p_{3}, p_{4}, p_{5}$ together with the points of the straight line joining $p_{1}$ and $p_{2}$, with all distances euclidean except the distance $p_{4} p_{5}$, which is defined to be the radius of the circumcircle of $p_{1}, p_{2}, p_{3}$, contains a subset $S$ of four distinct points $\left(p_{1}, p_{2}, x, y\right.$, where $x, y$ are any two points of the line other than $\left.p_{1}, p_{2}\right)$ such that every four points of $\Sigma$ with at least two points in common with $S$ are imbeddable in the plane $E_{2}$. But the space $\Sigma$ is obviously not imbeddable in $E_{2}$. 
A more striking way of lifting the demand that every $(n+2)$ tuple be imbeddable in $E_{n}$ is given by the following theorems.

THEOREM 1.2. Let $\Sigma$ be semimetric with at least $n+k+4$ distinct points. Then $\Sigma$ is imbeddable in $E_{n}$ if and only if $\Sigma$ contains at most $k$ free $(n+2)$-tuples.

Proof. We shall show that each $(n+2)$-tuple of $\Sigma$ is congruently contained in $E_{n}$.

Let, then, $p_{1}, p_{2}, \cdots, p_{n+2}$ be a free $(n+2)$-tuple. Since there are at most $k$ free $(n+2)$-tuples and at least $n+k+4$ points in $\Sigma$ it is readily seen that this $(n+2)$-tuple is contained in an $(n+5)$-tuple which has this $(n+2)$-tuple as its only free set of $n+2$ points. If $p_{1}, \cdots, p_{n+5}$ is such an $(n+5)$-tuple, each of the three sets of $n+4$ of these points obtained by omitting $p_{n}, p_{n+1}, p_{n+2}$ in turn have all $(n+2)$-tuples imbeddable in $E_{n}$, and hence the three $(n+4)$ tuples are themselves imbeddable in $E_{n}$. It follows that the rank of the determinant $D$ of the $n+5$ points is at most $n+2$, and so $D\left(p_{1}, p_{2}, \cdots, p_{n}, p_{n+1}, p_{n+2}\right)=0$.

Now each $n+1$ points of $\Sigma$ are imbeddable in $E_{n}$, for each $(n+1)$ tuple is contained in at least $k+3(n+2)$-tuples and not all of these are free since, by hypothesis, $\Sigma$ contains at most $k$ free $(n+2)$-tuples. Thus, each $n+1$ of the points $p_{1}, p_{2}, \cdots, p_{n+2}$ is imbeddable in $E_{n}$. Since the determinant $D$ of these $n+2$ points is zero, it follows that the points are imbeddable in $E_{n}$, and the theorem is proved.

The same kind of argument yields the next theorem.

TheOREM 1.3. Let $\Sigma$ be semimetric with power exceeding $\aleph_{0}$. Then $\Sigma$ is congruently contained in $E_{n}$ if and only if $\Sigma$ has at most $\aleph_{0}$ free $(n+2)$-tuples.

(II). The literature contains only two proofs of the quasi-congruence order $n+2$ property of the $E_{n}$, both of which are quite lengthy. A direct elementary proof is desired. Since the property is easily formulated in terms of determinant or quadratic form theory, attempts have been made to establish the result purely algebraically. For $n=1$, not a trivial case, a very simple proof has been found by the writer, but in general such efforts have so far not been effective. Since the complex space $K_{2}$ does not have quasi-congruence order 4 with respect to the class of complex distance spaces, a proof of the property for the $E_{n}$ must depend upon the reality of the field from which the distance elements are selected and hence is likely to be difficult. On the other hand, the writer has used algebraic methods to 
materially shorten the two earlier proofs. The question is, it seems, still open.

(III). It would be highly desirable to axiomatize the notion of quasi-congruence order or at least to express it in terms of more elementary properties of the space. A start in this direction has been made by $\mathrm{C}$. V. Robinson and the writer by showing that for complete, convex, externally convex metric spaces quasi-congruence order three (with respect to the class of semimetric spaces) is equivalent to the absence of equilateral triples of distinct points. This was done by establishing the following very simple metric characterization of the line [3].

Theorem 1.4. A complete, convex, externally convex metric space, with at least two points, is a straight line if and only if it does not contain an equilateral triple of distinct points.

This theorem has as immediate corollaries characterizations of the line diue to Lindenbaum and to Menger.

More recently the writer showed that a line segment is characterized among all compact and convex metric spaces by the absence of equilateral triples.

Quasi-congruence order $n+2$ readily implies the absence of equilateral $(n+2)$-tuples, but not conversely. The half-line, for example, has neither equilateral triples nor quasi-congruence order three, and the $K_{2}$ has neither equilateral quadruples (with nonzero side) nor quasi-congruence order 4 . What is a large class of spaces for which the two notions are logically equivalent?

In this connection, I would like to raise the question of interpreting topologically the absence of equilateral $k$-tuples. Biedermann has shown that a connected metric space in which each triple is linear (that is, a space strongly without equilateral triples) is homeomorphic with a line, a ray, or a segment. It was in fact this result that motivated Menger's characterization of euclidean subsets. (Menger showed that the homeomorphism of Biedermann is actually a congruence.)

If, now, one supposes merely that a connected metric space is without equilateral triples, what topological properties of the space ensue? Is a metric Peano continuum without equilateral triples an arc? If this is indeed the case (and I conjecture that it is) a topological property is given an elegant metric characterization. ${ }^{10}$ Such proble ms are part of a general program which seeks to determine what topologi-

${ }^{10}$ The writer has now established this conjecture. See New characterizations of segments and arcs, Proc. Nat. Acad. Sci. U. S. A. vol. 29 (1943) pp. 107-109. 
cal properties a space must possess in order that it may be homeomorphic to a space with given metric properties.

2. Congruence indices. Increasing study of characterization problems has yielded results that the concepts of congruence and quasicongruence orders are inadequate to describe. To meet this situation the notion of relative congruence indices was formulated [1]:

Let $\Gamma$ and $\Gamma^{*}$ be two given spaces and $\{\Delta\}$ a given class of spaces. The space $\Gamma$ has $\Gamma^{*}$-relative congruence indices $(n, k)$ with respect to the class $\{\Delta\}$ provided any space $\Delta$ of the class with more than $n+k$ distinct points is imbeddable in $\Gamma$ whenever each $n$ of its points (not necessarily distinct) is imbeddable in $\Gamma^{*}$.

The space $\Gamma^{*}$ is called $\Gamma$-catalytic of indices $(n, k)$ with respect to $\{\Delta\}$ since it (in general) facilitates the desired reaction of imbedding $\Delta$ in $\Gamma$ without participating in the result, for $\Delta$ is not necessarily imbeddable in $\Gamma^{*}$. If $\Gamma \equiv \Gamma^{*}$ the indices $(n, k)$ are called congruence indices of $\Gamma$ with respect to the class $\{\Delta\}$.

It is easy to see that congruence and quasi-congruence orders correspond to congruence indices $(k, 0)$ and $(k, 1)$, respectively, and that if $\Gamma$ has $\Gamma^{*}$-relative congruence indices $(n, k)$, then $\Gamma$ has $\Gamma^{*}$-relative indices $\left(n^{\prime}, k^{\prime}\right)$ if $n \leqq n^{\prime}$ and $n+k \leqq n^{\prime}+k^{\prime}$, the class of comparison spaces being fixed. We order these indices lexicographically, and call those indices $(n, k)$ "best" which are not preceded by any indices $\left(n^{\prime}, k^{\prime}\right)$. It is important to observe that this ordering is not that of logical implication, for with respect to the latter criterion the indices do not form an ordered set. Thus a two-dimensional spherical cap of radius less than $\pi r / 2$ has, with respect to the class of all semimetric spaces, congruence indices $(4,2)$ and also indices $(5,0)$, but neither set logically implies the other.

Keeping the comparison class of spaces fixed, let the best congruence indices of a space $\Gamma$ be $(n, k)$, with $\left(n^{\prime}, k^{\prime}\right)$ the best $\Gamma^{*}$-relative congruence indices. The space $\Gamma^{*}$ is strongly or weakly catalytic according as $n^{\prime}<n$ or $n^{\prime}=n, k^{\prime}<k$, respectively. In case the indices $\left(n^{\prime}, k^{\prime}\right)$ are no better than $(n, k), \Gamma^{*}$ is formally catalytic, while if $n^{\prime}<n$ and $k^{\prime}<k, \Gamma^{*}$ is perfectly catalytic. An open hemisphere of $S_{2, r}$, for example, is a strongly catalytic space for $S_{2, r}$, since by its use the best congruence indices $(5,0)$ of $S_{2, r}$ with respect to semimetric spaces are bettered by the hemisphere-relative congruence indices $(4,2)$. In fact, it has been shown that the $S_{n, r}{ }^{11}$ has $\Gamma^{*}$-relative congruence indices $(n+2, n)$ with respect to all semimetric spaces,

${ }^{11} S_{n, r}$ denotes the $n$-dimensional surface of a sphere of radius $r$ in $E_{n+1}$, with geodesic (shorter great circle arc) metric. 
where $\Gamma^{*}$ denotes an open ( $n$-dimensional) hemisphere of the $S_{n, r}$.

It is worth noting that pseudo-euclidean spaces fall under the notion of catalytic spaces. Clearly every subset of a catalytic space is catalytic (with the same indices) but the interest lies in enlarging rather than depleting these spaces, and the problem of finding saturated catalytic spaces of given indices arises.

The writer recently proposed the problems of finding the best congruence indices of the (closed) $n$-dimensional hemisphere and of small $n$-dimensional spherical caps with respect to (1) the class of semimetric spaces and (2) the set of all subsets of the containing $S_{n, r}$. The second inquiry led to posing the following question: for what integer $k$ does the intersection of each $k$ members of a family of convex subsets of the $n$-dimensional spherical surface $S_{n, r}$ imply the existence of a common point for the family?

In his Missouri dissertation, C. V. Robinson investigated these problems [9]. He showed that the best congruence indices of the $n$ dimensional hemisphere with respect to semimetric spaces are $(2 n+1,1)$. Even with respect to subsets of the containing $S_{n, r}$ these indices cannot be bettered. It follows that any subset $S$ of $S_{n, r}$ is coverable by a hemi- $S_{n, r}$ provided $S$ contains more than $2 n+2$ distinct points and each $2 n+1$ of its points is coverable by the hemi$S_{n, r}$.

Of particular interest is the plane analogue of a cap theorem to the effect that a circular disc will cover a plane set $P$ if and only if each three points of $P$ are coverable by the disc. This behavior of the circular disc is all the more striking when contrasted with that of other convex subsets of the plane such as the square or elliptical disc. For neither of these figures does any such integer $k$ exist; for example, for any integer $k$ however large there are plane sets not coverable by a square (elliptical) disc even though each $k$ points of the set are so coverable. The same is true for any portion of the plane bounded by a broken-line curve. The possession of indices $(3,0)$ with respect to subsets of the plane has been made the basis of a characterization of the circular disc [8].

TheOREM 2.1. The circular disc is the only connected, simply connected domain (closure of a bounded open set) of the plane with congruence indices $(3,0)$ with respect to plane sets.

It would be highly desirable to make an exhaustive classification of plane sets according to their congruence indices with respect to subsets of the plane. The problems involved seem quite difficult and only the beginnings of a systematic investigation have been made. With 
respect to the class of linear subsets we have a partial enumeration of linear sets with fixed congruence orders (finite, hyperfinite, and transfinite). ${ }^{12}$ Investigations aimed at completing these results and developing a similar theory for plane subsets are now in progress.

In this connection interest is attached to a new type of problem recently considered which I illustrate here for the simplest case. It is easily seen that corresponding to each integer $k$ there exists a bounded and closed subset of $E_{1}$ with best (finite) congruence order exceeding $k$. Thus if $P$ is an arbitrary bounded and closed linear set, one may not assert that any linear set $Q$ is imbeddable in $P$ whenever each $k$ of its points are, even for $k$ arbitrarily large. Note that, as is usual in imbedding theorems, the condition "every $k$ points of $Q$ are imbeddable in $P$ " is a unilateral one. Suppose, on the other hand, it is also assumed that every $k$ points of $P$ are imbeddable in $Q$. Then one easily proves that $P$ and $Q$ are congruent when $k=4$; that is, if $P$ and $Q$ are linear sets with $P$ or $Q$ bounded and closed, then $P \approx Q$ if and only if each four points of $P$ are imbeddable in $Q$ and each four points of $Q$ are imbeddable in $P$. This bilateral type of condition gives rise to several new problems and causes the notion of congruence order (based upon unilateral conditions) to be viewed in a somewhat different light.

In answering the question raised concerning intersections of convex subsets of $S_{n, r}$, the following theorems were obtained. ${ }^{13}$

TheOREM 2.2. If each $n+k+2$ members of a family of convex subsets of the sphere $S_{r, r}$ intersect, and if one member contains no $S_{k, r}$ $(0 \leqq k \leqq n)$, then there is a point common to all.

TheOREM 2.3. A family of more than $2 n+2$ convex subsets of $S_{n, r}$ has a common point if each $2 n+1$ of them intersect.

Additional theorems are obtained when the diameters of the convex subsets are subject to certain restrictions.

${ }^{12}$ A space $\Sigma$ has hyperfinite congruence order with respect to a class of spaces $\{\Delta\}$ if and only if each member of the class is imbeddable in $\Sigma$ whenever each of its finite subsets are. Thus hyperfinite congruence order is intermediate between finite and transfinite congruence orders. Each self-compact linear set has hyperfinite congruence order with respect to semimetric spaces; examples have been given of linear sets with hyperfinite and no finite congruence order, and transfinite but not hyperfinite congruence order. The complement of a finite or denumerable linear set has best congruence order $c$ (the power of the continuum). These notions were introduced in the Missouri dissertation of C. V. Robinson.

${ }^{13}$ A subset of $S_{n, r}$ is convex provided it is a product of hemispheres. This definition rules out the whole $S_{n, r}$ and includes a pair of diametral points. Thus from Theorem 2.2 it follows that a family of convex subsets of $S_{n, r}$ has a common point whenever each $2 n+2$ of them intersect. 
Intersection theorems for convex subsets of $E_{n}$ were obtained by Helly. Due to the variety of results such problems on the sphere turn out to be more interesting than their analogues in euclidean spaces. In contrast, for example, to the Helly theorem in $E_{2}$ which states that any family of convex subsets has a non-empty product whenever each three members of the family intersect, it is found that a family of convex subsets of $S_{2, r}$ have a common point if each six have or (if the family has more than six members) if each five have, or (if one member of the family contains no diametral point-pairs) if each four have. If each of the convex subsets has spherical diameter less than $2 \pi r / 3$ it suffices for each three of the sets to have a common point. It is clear how these results may be translated into "covering" theorems for spherical caps.

3. $\delta$-supplementation and elliptic space. If $\Sigma$ is semimetric with finite diameter $d$, denote the set of unordered pairs of its elements by $S^{2}$, and let $S^{2}=S_{1}+S_{2}, S_{1} \cdot S_{2}=0$, be any decomposition of $S^{2}$ into two mutually exclusive subsets. Corresponding to such a decomposition, a real, non-negative function $F(x)$ is defined:

$$
\begin{array}{ll}
F(x)=x, & x=p q ;(p, q) \in S_{1}, \\
F(x)=\delta-x, & x=p q ;(p, q) \in S_{2},
\end{array}
$$

where $\delta$ is a fixed real number not less than $d$. Transforming metrically the space $\Sigma$ by this function $F$ yields a space which is in general not semimetric. ${ }^{14}$ Nor does it necessarily become semimetric upon identification of those points $p, q$ (and only those) with $p q^{*}=F(p q)$ $=0$, for such an identification is justified (keeping to a single-valued metric) if and only if for each element $r$ of the space $p r^{*}=q r^{*}$.

If, however, $\delta>d$, such an identification does convert the transformed space into a semimetric one whose point set may, on account of the identification, differ from that of $\Sigma$. We denote such a space by $\Sigma^{*}$ and refer to it as a $\delta$-supplement of $\Sigma$ (more precisely, the $\delta_{F}$-supplement of $\Sigma$ ). We write $\Sigma^{*}=\sup _{\delta} \Sigma$ when there is no need to specify the function $F$.

An important example of a $\delta$-supplement is obtained by taking the

${ }^{14}$ A space $F(\Sigma)$ which arises from $\Sigma$ by replacing the metric $p q$ of $\Sigma$ by $F(p q)$, where $F$ is a real, single-valued, non-negative function defined for every $x=p q$, $p, q \in \Sigma$, is called the metric transform of $\Sigma$ by $F$. A study of metric transforms was begun by the writer in 1934 (Ergebnisse eines der Mathematischen Kolloquiums, Wien, no. 7, pp. 8-10). Metric transforms of euclidean spaces into subsets of Hilbert space have been extensively investigated by Schoenberg and von Neumann $(1938,1942)$. 
$n$-dimensional convex spherical surface $S_{n, r}$ as $\Sigma$, and transforming it metrically by the function $F$.

$$
\begin{array}{ll}
F(x)=x, & x=p q ; p q \leqq \pi r / 2, \\
F(x)=\pi r-x, & x=p q ; p q>\pi r / 2 .
\end{array}
$$

(Here $\delta=d$.) The resulting semimetric space is the $n$-dimensional elliptic space $\mathcal{E}_{n, r}$ with space constant $r$.

In work still in progress, the notion of $\delta$-supplementation is made the basis for characterizing subsets of $\varepsilon_{n, r}$ among all semimetric spaces [2]. Elliptic space differs profoundly (both metrically and topologically) from all spaces heretofore treated and these differences make the older methods either inapplicable or extremely tedious. Fundamental in our work is the following theorem.

THEOREM 3.1. A semimetric $\Sigma$ is congruently contained in $\varepsilon_{n, r}$ if and only if (i) no distance in $\Sigma$ exceeds $\pi r / 2$ and (ii) there exists at least one function $F$ such that the $\delta_{F}$-supplement of $\Sigma$ is imbeddable congruently in $S_{n, r}$, where $\delta=\pi r$.

PROOF. Let $\Sigma$ be congruently contained in $\varepsilon_{n, r}$. Then evidently $p q \leqq \pi r / 2$ for each pair $p, q$ of elements of $\Sigma$. Denoting the metric transform of $S_{n, r}$ by the function $F$ defined in ( $\dagger$ ) by $\sup _{\pi r} S_{n, r}$, we have $\varepsilon_{n, r}=\sup _{\pi r} S_{n, r}$. Evidently

$$
\text { subset } \varepsilon_{n, r}=\operatorname{subset}\left(\sup _{\pi r} S_{n, r}\right)=\sup _{\pi r}\left(\text { subset } S_{n, r}\right) \text {, }
$$

and hence there exists a $\sup \left(\operatorname{subset} \varepsilon_{n, r}\right.$ ) which is a subset of $S_{n, r}$. Since, now, $\Sigma$ is congruently contained in $\varepsilon_{n, r}$, there is a subset of $\varepsilon_{n, r}$ congruent with $\Sigma$, and, by the above, a supplement of this subset is a subset of $S_{n, r}$. Thus, a supplement of $\Sigma$ is imbeddable in $S_{n, r}$ and the necessity is proved.

On the other hand suppose that $\Sigma$ is semimetric with distances not exceeding $\pi r / 2$ and that some $\pi r$-supplement of $\Sigma$ is congruently contained in $S_{n, r}$. Denote any such supplement by sup $\Sigma$. Then there exists a subset $S_{n, r}^{\prime}$ of $S_{n, r}$ such that $\sup \Sigma$ is congruent with $S_{n, r}^{\prime}$. Now $\sup _{\pi r} S_{n, r}^{\prime}=$ subset $\varepsilon_{n, r}$ and hence $\sup _{\pi r}(\sup \Sigma)$ is congruently contained in $\varepsilon_{n, r}$.

The proof is complete when it is shown that for every sup $\Sigma$, $\sup _{\pi \imath}(\sup \Sigma)$ is identical with $\Sigma$. To this end, denote distances in sup $\Sigma$ by $d(p, q)$ and in $\sup _{\pi r}(\sup \Sigma)$ by $d_{\pi r}(p, q)$. The same argument shows that the two pointsets and the two distance-sets are identical, for let $p=q$ in $\Sigma$. Then $p q=0$. If $d(p, q)=0$, then $d_{\pi r}(p, q)=0$, while if $d(p, q)=\pi r$, then $d_{\pi r}(p, q)=0$. Hence $p=q$ in $\Sigma$ implies $p=q$ in 
$\sup _{\pi r}(\sup \Sigma)$. Finally, if $p$ and $q$ are distinct points of $\Sigma$, then $0<p q$ $\leqq \pi r / 2$. If $d(p, q)=p q$ then, since $p q \leqq \pi r / 2, d_{\pi r}(p, q)=p q>0$; while if $d(p, q)=\pi r-p q \geqq \pi r / 2$, then $d_{\pi r}(p, q)=\pi r-(\pi r-p q)=p q>0$, and the theorem is proved.

By virtue of this theorem, imbedding in $\varepsilon_{n, r}$ is referred to imbedding $\pi r$-supplements of semimetric spaces in $S_{n, r}$. In any such supplement the distance $p q$ of two points of $\Sigma$ is either unchanged or replaced by $\pi r-p q$, and hence $\cos (d(p, q) / r)$ differs from $\cos (p q / r)$ at most in sign. Applying known imbedding theorems for the $S_{n, r}$ we have the following theorem.

Theorem 3.2. A semimetric m-tuple $p_{1}, p_{2}, \cdots, p_{m}$ is imbeddable in $\varepsilon_{n, r}$ if and only if (i) $0<p_{i} p_{j} \leqq \pi r / 2(i, j=1,2, \cdots, m ; i \neq j)$ and (ii) there exists an m-rowed symmetric square matrix $\epsilon=\left(\epsilon_{i j}\right), \epsilon_{i j}= \pm 1$, $\epsilon_{i i}=1(i, j=1,2, \cdots, m)$ such that the determinant $\left|\epsilon_{i j} \cos \left(p_{i} p_{j} / r\right)\right|$ $(i, j=1,2, \cdots, m)$ has rank not exceeding $n+1$, with all nonvanishing principal minors positive.

A determinant (symmetric) satisfying the condition (ii) of the theorem is said to have positive $\epsilon$-rank not exceeding $n+1$.

Now it is easy to show that each separable semimetric space is congruently contained in $\varepsilon_{n, r}$ whenever each of its finite subsets has this property, and hence we have obtained necessary and sufficient conditions for the imbedding of any separable semimetric space in a given $n$-dimensional elliptic space. It is desirable, however, to establish reduction theorems which express the imbedding in terms of the congruent mapping of subsets of a fixed number of points. Such theorems hinge upon the following query.

QUERy. What is the smallest integer $k$ such that the determinant $\left|\cos \left(p_{i} p_{j} / r\right)\right|(i, j=1,2, \cdots, m)$ has positive $\epsilon$-rank not exceeding $n+1$ whenever each of its principal minors of order $k$ has positive $\epsilon$-rank not exceeding $n+1$ ?

It is conjectured that $k=n+3$, but so far this has been proved only for $n=1 .^{15}$ Thus any separable semimetric space is imbeddable in $\varepsilon_{1, r}$ if and only if each four of its points have this property. This can be established in other ways, and without the hypothesis of separability. But for $n>1$, the algebraic method discussed here appears to be the only feasible approach. The above conjecture means (if verified)

\footnotetext{
${ }^{15}$ Examples show that $k$ is not less than $n+3$.
} 
that $\varepsilon_{n, r}$ has (like euclidean, hyperbolic and spherical spaces of $n$ dimensions) congruence order $n+3$. It would be interesting if the different topological properties of $\varepsilon_{n, r}$ would be made manifest by a difference in the congruence order.

4. Concluding remarks. In conclusion it should be observed that this paper has been concerned with completely global imbeddings and characterizations though interesting results in which local properties are assumed have also been obtained. A completely local type of theorem is one characterizing the line segment among all arcs of a metric ptolemaic space by the vanishing of the metrically defined Menger curvature at each point, while Gauss surfaces have been characterized among all compact and convex metric spaces by the existence at each point of a metrically defined surface curvature. A different ("mixed") type of imbedding theorem is expressed in terms of the local property of imbedding neighborhoods in $E_{n}$ together with the global condition that each $n+3$ points be congruently contained in some euclidean space.

It has not been possible to present here any of the applications of metric imbedding theorems to other domains. Metric methods have already been successfully applied to many parts of analysis and algebra as well as to geometry, and there is reason to believe that continued cultivation of this field will abundantly increase both the number and the importance of these applications.

\section{REFERENCES}

1. L. M. Blumenthal, $A$ new concept in distance geometry with applications to spherical subsets, Bull. Amer. Math. Soc. vol. 47 (1941) pp. 435-443.

2. - Metric characterization of n-dimensional elliptic space $\varepsilon_{n, r}$, Bull. Amer. Math. Soc. abstract 48-1-75.

3. L. M. Blumenthal and C. V. Robinson, $A$ new characterization of the straight line, Reports of a Mathematical Colloquium, issue 2 (1940) pp. 25-27.

4. B. E. Gillam, $A$ new set of postulates for euclidean geometry, Revista de Ciencias vol. 42 (1940) pp. 869-899.

5. K. Menger, Some applications of point-set methods, Ann. of Math. (2) vol. 32 (1931) pp. 739-760. See in particular pp. 751, 752.

6. - Untersuchungen über allgemeine Metrik, I, II, III, Math. Ann. vol. 100 (1928) pp. 75-163. The Zweite Untersuchung is devoted to the characterization of euclidean subsets and spaces.

7. P. M. Pepper, Nearly euclidean imbedding spaces for pseudo n-sets, Reports of a Mathematical Colloquium, issue 3 (1941) pp. 34-46. See also issue 2 (1940), pp. 28-32.

8. C. V. Robinson, A characterization of the disc, Bull. Amer. Math. Soc. vol. 47 (1941) pp. $818,819$. 
9. - Spherical theorems of Helly type and congruence indices of spherical caps, Amer. J. Math. vol. 64 (1942) pp. 260-272.

10. I. J. Schoenberg, Remarks to Maurice Fréchet's article "Sur la défnition axiomatique d'une classe d'espaces vectoriels distanciés applicables vectoriellement sur l'espace de Hilbert," Ann. of Math. (2) vol. 36 (1935) pp. 724-732.

The University OF MisSOURI 